\title{
Culturing bone marrow cells with dexamethasone and ascorbic acid improves osteogenic cell sheet structure
}

\section{Akahane, \\ T. Shimizu, \\ T. Kira, \\ T. Onishi, \\ Y. Uchihara, \\ T. Imamura, \\ Y. Tanaka}

Nara Medical

University Faculty of

Medicine, Nara, Japan

\section{Objectives}

To assess the structure and extracellular matrix molecule expression of osteogenic cell sheets created via culture in medium with both dexamethasone (Dex) and ascorbic acid phosphate (AscP) compared either Dex or AscP alone.

\section{Methods}

Osteogenic cell sheets were prepared by culturing rat bone marrow stromal cells in a minimal essential medium (MEM), MEM with AscP, MEM with Dex, and MEM with Dex and AscP (Dex/AscP). The cell number and messenger $(m)$ RNA expression were assessed in vitro, and the appearance of the cell sheets was observed after mechanical retrieval using a scraper. $\beta$-tricalcium phosphate ( $\beta$-TCP) was then wrapped with the cell sheets from the four different groups and subcutaneously implanted into rats.

\section{Results}

After mechanical retrieval, the osteogenic cell sheets from the MEM, MEM with AscP, and MEM with Dex groups appeared to be fragmented or incomplete structures. The cell sheets cultured with Dex/AscP remained intact after mechanical retrieval, without any identifiable tears. Culture with Dex/AscP increased the mRNA and protein expression of extracellular matrix proteins and cell number compared with those of the other three groups. More bridging bone formation was observed after transplantation of the $\beta$-TCP scaffold wrapped with cell sheets cultured with Dex/AscP, than in the other groups.

\section{Conclusions}

These results suggest that culture with Dex/AscP improves the mechanical integrity of the osteogenic cell sheets, allowing retrieval of the confluent cells in a single cell sheet structure. This method may be beneficial when applied in cases of difficult tissue reconstruction, such as nonunion, bone defects, and osteonecrosis.

Cite this article: Bone Joint Res 2016;5:569-576.

Keywords: Osteogenesis; Cell sheet; Mechanical retrieval

\section{Article focus}

- This study focused on the mechanical retrieval technique to create osteogenic cell sheets.

\section{Key messages}

- Culture with dexamethasone/ascorbic acid phosphate improves the mechanical integrity of osteogenic cell sheets, allowing retrieval of confluent cells in a cell sheet structure.

\section{Strengths and Limitations}

doi: $10.1302 / 2046-3758.511$ BJR-2016-0013.R1

Bone Joint Res 2016;5:569-576. Received: 21 January 2016; Accepted: 9 September 2016
- Strength: this method may be beneficial for cases of difficult tissue reconstruction, such as nonunion and bone defects.
- Limitations: Future studies should focus on human bone marrow stromal cells in order to determine if culture with Dex/ AscP improves the integrity of osteogenic cell sheets.

\section{Introduction}

Mesenchymal stem cells (MSCs) are adult stem cells which can be isolated from tissues such as bone marrow, ${ }^{1,2}$ adipose, ${ }^{3}$ and skeletal muscle. ${ }^{4}$ Because MSCs are multipotent and can differentiate into osteoblasts, 2,5 chondrocytes, ${ }^{6}$ and neurons, 7,8 they have been used in regenerative medicine to treat 
various diseases and tissue injuries, 9,10 including, tissue engineering using an in vitro cell culture. Bone marrow stromal cells (BMSCs) are commonly used for clinical treatment ${ }^{2,6}$ because they can be harvested from a patient's pelvis by needle aspiration, a minimally invasive procedure compared with harvesting bone. Therefore, BMSC populations containing MSCs are a good source for creating tissue-engineered bone. ${ }^{11,12}$

For clinical applications, the BMSCs harvested from patients are expanded in vitro, and then seeded onto scaffolding materials such as hydroxyapatite $(H A)^{13}$ and $\beta$-tricalcium phosphate ( $\beta$-TCP). ${ }^{14}$ When scaffolds seeded with BMSCs are transplanted into patients, more cells that are seeded on the scaffolds at implantation result in better bone formation. BMSCs are able to differentiate into osteoblastic lineage cells after subculture in osteogenic induction media containing dexamethasone (Dex), ascorbic acid phosphate (AsCP), and $\beta$-glycerophosphate $(\beta-G P) .{ }^{13,15,16}$ Combinations of BMSCs with a scaffolding material, such as HA and $\beta$-TCP, promote the formation of new bone tissue in vivo following subcutaneous transplantation, both when using freshly isolated bone marrow cells ${ }^{5}$ and culture-expanded BMSCs. ${ }^{2,17-19}$

Many recent studies have reported methods for creating cell sheets including thermo-responsive polymergrafted culture dishes 20,21 and mechanical retrieval. ${ }^{22-24}$ We have previously proposed solutions to undertake difficult tissue regeneration, including fracture nonunion ${ }^{25}$ and ligament reconstruction ${ }^{26}$ using scaffold-free cell sheet transplantation. We have also reported a technique for cell sheet injection we call 'injectable bone', that promotes osteogenesis in necrotic bone and implanted materials. ${ }^{24,27}$ The method we previously reported to create osteogenic matrix cell sheets from BMSCs requires culturing the cells with both Dex and AscP to create confluent cells in the structure of sheets that can be mechanically retrieved. ${ }^{22,27}$ In our previous reports, however, we did not determine whether culture of the cells with Dex or AscP alone induces sufficient structure to allow for the mechanical retrieval of the confluent cells in the structure of sheets. Therefore, in the present study, we used BMSCs obtained from rat bone marrow and assessed the structure and expression of extracellular matrix (ECM) components of the cell sheets created by culturing in medium with both Dex and AscP, compared with those cultured with either Dex or AscP alone.

\section{Materials and Methods}

Experimental materials. Male seven-week-old Fischer rats were purchased from Japan SLC Inc. (Shizuoka, Japan). Minimal essential medium (MEM), antibiotics $(100 \mathrm{U} / \mathrm{mL}$ penicillin and $100 \mu \mathrm{g} / \mathrm{mL}$ streptomycin) and Trypsin/ethylenediaminetetraacetic acid (EDTA) were purchased from Nacalai Tesque (Kyoto, Japan). Culture flasks and plates were from Corning (Corning, New York, New York), and fetal bovine serum (FBS) and phosphate-buffered saline (PBS) from Gibco (Life Technologies, Carlsbad, California). AscP was also purchased from Wako Pure Chemical Industrials (Kyoto, Japan). Dex was purchased from Sigma (St. Louis, Missouri). Primer and probe sets and reagents for realtime quantitative polymerase chain reaction (PCR) were purchased from Applied Biosystems (Norwalk, Connecticut). $\beta$-TCP granules (OSferion $60,60 \%$ porosity) were purchased from Olympus (Tokyo, Japan), and RNeasy Mini Kits were purchased from QIAGEN Inc. (Venlo, The Netherlands).

Preparation of the bone marrow stromal cells. BMSCs were prepared as previously reported.22,25 Briefly, BMSCs were obtained from the femur shafts of male seven-week-old Fischer rats. Male rats were chosen as they are larger than females and are therefore easier to handle. Both ends of each femur were removed from the epiphysis and the marrow was flushed out using $10 \mathrm{~mL}$ of standard culture medium, which consisted of MEM containing 15\% FBS and antibiotics, expelled from a syringe through a 21-gauge needle. The obtained cells were collected in two T-75 flasks containing the standard culture medium. Once they were confluent, the cells were released from the culture substratum using trypsin/EDTA. In the present study, BMSCs were prepared from three different donor rats because the characteristics of BMSCs from primary culture may vary among each individual.

In vitro cell culture. The released BMSCs were seeded at a density of $1 \times 10^{4}$ cells $/ \mathrm{cm}^{2}$ in order to assess cell sheet formation by macroscopic and microscopic observation. The cells were cultured with the standard medium alone (MEM group), the standard medium with $0.28 \mathrm{mM}$ AscP (Ascp group), the standard medium with $10 \mathrm{nM}$ Dex (Dex group), or the standard medium with $10 \mathrm{nM}$ Dex and $0.28 \mathrm{mM}$ AscP (Dex/AscP group) for 14 days. The BMSCs were cultured in a humidified atmosphere of $95 \%$ air with $5 \% \mathrm{CO}_{2}$ at $37^{\circ} \mathrm{C}$ in 96 -well plates for the cell proliferation assay, and 6-well plates for the assessment of ECM component expression and Western blotting. Macroscopic and microscopic observations were made of cells cultured in $100 \mathrm{~mm}$ dishes, by eye.

MTT assay. The cell proliferation of each group was measured using the MTT assay (3-(4,5-dimethylthiazol2-yl)-5-(3-carboxymethoxyphenyl)-2-(4-sulfophenyl)$2 \mathrm{H}$-tetrazolium). Cells were seeded onto 96-well culture plates $\left(1 \times 10^{4}\right.$ cells $\left./ \mathrm{cm}^{2}\right)$ and cultured with $100 \mu \mathrm{L}$ of medium from each group for five days $(n=5)$. Then, the cell proliferation was determined using CellTiter 96 Aqueous One Solution Reagent (Promega, Madison, Wisconsin). Once the medium was removed and the cells were washed with PBS, $100 \mu \mathrm{L}$ of medium and $20 \mu \mathrm{L}$ of MTS solution were added per well and incubated for one hour at $37^{\circ} \mathrm{C}$. Finally, formazan absorbance at $490 \mathrm{nM}$ was measured in a microplate reader. The experiments in each group were performed in triplicate. 
RNA isolation and real-time quantitative PCR. The gene expression levels of collagen types I, laminin, fibronectin and osteocalcin were measured in each group. Total RNA was isolated from each group using RNeasy Mini RNA extraction kits. Cultured cells from each group in 6-well plates were rinsed twice with PBS and then treated with $350 \mu \mathrm{L}$ of the isolation reagent and converted to complementary (c)DNA following the manufacturer's protocol. To measure the messenger (m)RNA expression levels, real-time quantitative PCR (ABI StepOne Plus Real Time PCR System), using primers for rat CDNAs, as previously described. ${ }^{24}$ The target mRNA levels were compared after normalising to glyceraldehyde-3-phosphate dehydrogenase (GAPDH) mRNA levels as an internal standard that corrected for differences in the efficiency of reverse transcription between samples. The primers for the target mRNAs were collagen type I (Rn00801649 g1), laminin (Rn00564264 m1), fibronectin (Rn00569575 m1), osteocalcin (Rn01455285 g1) and GAPDH (Rn99999916 s1). The thermal cycling conditions were 20 seconds at $95^{\circ} \mathrm{C}$ for activation of the TaqMan Fast Universal PCR Master Mix, followed by 40 cycles of 1 second at $95^{\circ} \mathrm{C}$ for denaturing and 20 seconds at $60^{\circ} \mathrm{C}$ for annealing and extension. The experiments were performed in triplicate.

Western blotting. Secretion of ECM components was evaluated by Western blot analysis. Cell lysates were prepared using mammalian protein extraction reagent (M-PER; Pierce, Rockford, Illinois) containing protease inhibitor cocktails of $1 \mathrm{mM}$ sodium orthovanadate and $25 \mathrm{mM} \mathrm{NaF}$. The protein concentration was measured twice using a BCA Protein Assay Kit (Pierce) with bovine serum albumin as the standard. Cell lysate samples containing $10 \mu \mathrm{g}$ of protein were separated by SDSpolyacrylamide gel electrophoresis and transferred to a polyvinylidene difluoride membrane (Invitrogen, Thermo Fisher Scientific, Waltham, Massachusetts). The membrane was incubated with antibodies against collagen type I (Rockland Immunochemicals Inc., Pottstown, Pennsylvania), fibronectin (Rockland Immunochemicals), laminin (Santa Cruz Biotechnology, Dallas, Texas) and actin (Merck MILLIPORE, Darmstadt, Germany) diluted in phosphate buffered saline (PBS-T) with 5\% non-fat dry milk at $4^{\circ} \mathrm{C}$ overnight. The membrane was then washed in PBS-T and incubated with appropriate secondary antibodies at room temperature for one hour. The immune complexes were made visible with a chemiluminescent substrate (Western Lightning Plus-ECL; PerkinElmer, Waltham, Massachusetts). The signal intensities of the expressed protein bands were measured using densitometric analysis (Image) software; National Institute for Health, Bethesda, Massachusetts) and normalised to the ratio of the band densities in the MEM group, which was arbitrarily defined (by MA) as one unit.

Measurement of proteoglycan (PG) content. PG in the cell matrix of each group was measured. Briefly, cells of each group were cultured in 6-well plates $(n=3)$ and PG in the cell matrix at day 14 of each group was measured by ELISA (Proteoglycan Detection Kit, Astarte Biologics, Bothell, Washington). This experiment was performed in triplicate. Subcutaneous transplantation of the composite of $\boldsymbol{\beta}$-TCP granules and cultured cells. To assess the in vivo bone formation pattern, the cultured cells from all four groups were scraped off and transplanted together with $\beta$-TCP granules $(n=3)$. Briefly, the $\beta$-TCP scaffolds were combined with the cultured cells and then subcutaneously implanted into the backs of the eight rats. ${ }^{28}$ The $\beta$-TCP scaffolds were harvested after four weeks, fixed in $10 \%$ neutral-buffered formalin, decalcified with EDTA solution, and embedded in paraffin. The specimens were cut through the middle and stained with haematoxylin $(\mathrm{H})$ and eosin (E). The experiments were performed in duplicate. Two of the authors (MA and TS) observed the H-E sections to assess whether there was bone tissue. MA knew the sample names, however TS was blinded. The authors got same result.

Osteogenesis at the bone defect site. To assess whether the complete cell sheet (cultured in Dex/AscP medium) could form bone tissue at the bone defect site, another animal study was performed. Briefly, femurs harvested from donor rats were irradiated (60 Gray) to obtain dead bone. ${ }^{29}$ Then, a bone defect was created at the shaft of the dead femur, and the defect was filled with $\beta$-TCP granules combined with cell sheets. Subsequently, the dead femur with $\beta$-TCP granules/cell sheet was subcutaneously implanted into the backs of rats. As a control, dead femur, in which the bone defect was filled with $\beta$ TCP granules alone, was transplanted subcutaneously. The femurs were harvested after four weeks, fixed in 10\% neutral-buffered formalin, decalcified with EDTA solution, and embedded in paraffin. The specimens were cut through the longitudinal axis and stained with $\mathrm{H}$ and $\mathrm{E}$. Two of the authors (MA and TS) observed the H-E sections to assess whether there was bone tissue. MA knew the sample names, however TS was blinded. The authors got same result.

Statistical analysis. This was performed in SPSS (Version 22, IBM, Armonk, New York). The statistical significance of differences between groups was determined using one-way analysis of variance with Tukey post hoc multiple comparisons tests. A p-value of $<0.05$ was considered statistically significant.

\section{Results}

The appearance of the retrieved cell sheets in each group after scraping are shown in Figure 1. No obvious tears in the sheet structure of the Dex/AscP group were seen by macroscopic observation (Fig. 1a), and those cell sheets were easily removed from the wells. In contrast, the cell sheets in the MEM, AscP, and Dex groups appeared as fragments and incomplete structures. Tears and fragmentation of the sheet structure was found by microscopic observation (Fig. 1b) in the MEM, AscP, and Dex groups. In contrast, the Dex/AscP 

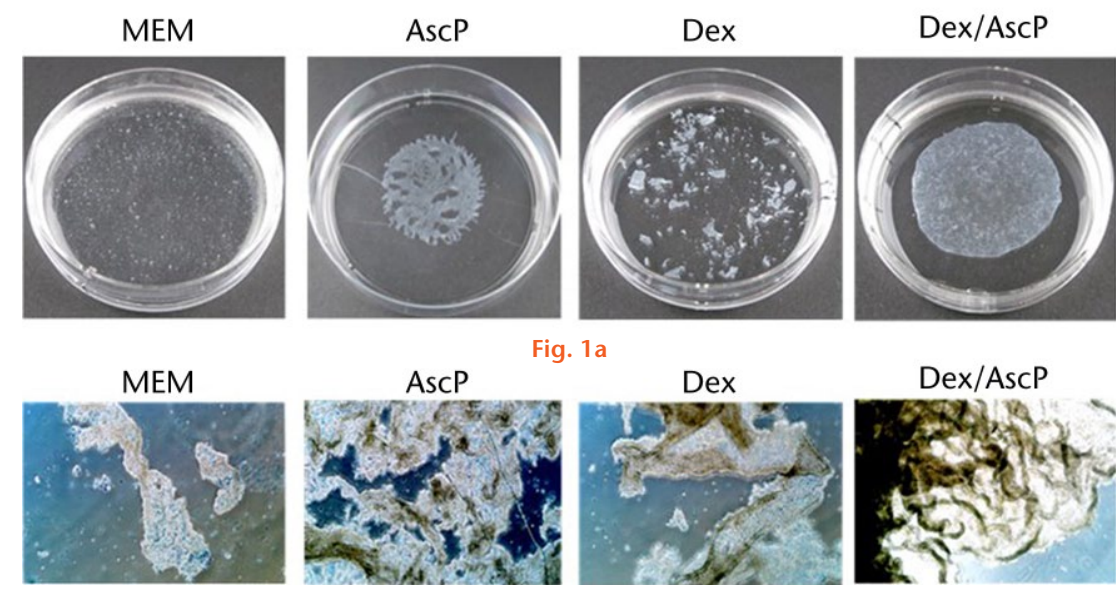

Dex/AscP

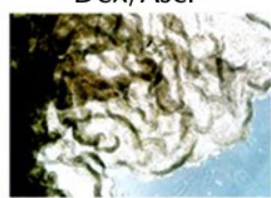

Fig. $1 b$

Macroscopic (a) and microscopic (b) views of the retrieved cell sheets.

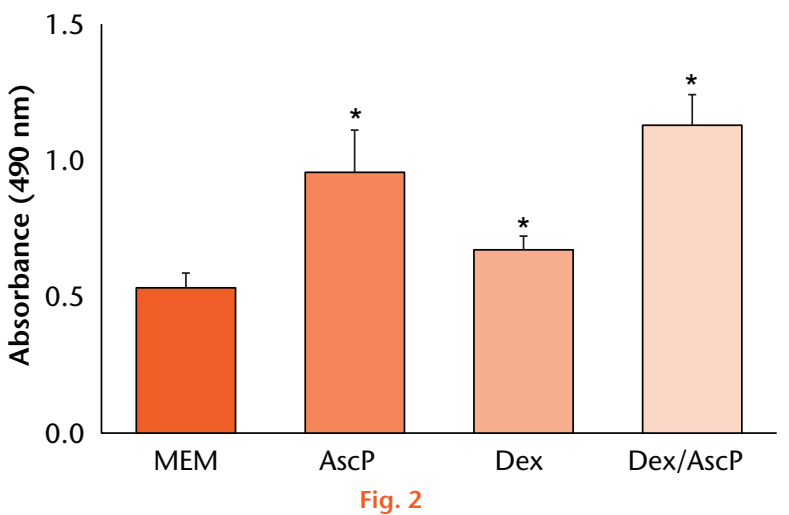

MTT assay of the bone marrow stromal cells in groups ( ${ }^{*} \mathrm{p}$-values (versus MEM): are AscP = 8.0 $\times 10^{-6}$; Dex $=0.317$; Dex $/$ AscP $=3.8 \times 10^{-8}$, respectively).

group contained sheet folds without any tears and only a few cells in the culture medium around the sheet, indicating that culture with Dex/AscP allows for the mechanical retrieval of confluent cells in a single sheet structure.

The results of the MTT assay are shown in Figure 2. The number of the cells in the AscP and Dex/AscP groups were significantly higher than that in the MEM group ( $p$-values (versus MEM) are AscP $=8.0 \times 10^{-6}$, Dex $=0.317$ and Dex/AscP $=3.8 \times 10^{-8}$, respectively). The highest cell number at day 14 was observed in the Dex/AscP group.

The real time PCR assessments of the mRNA expression levels of the ECM components are shown in Figure 3. The expressions of collagen types I and laminin were significantly higher in the Dex/AscP group than in the MEM group ( $p=0.001$ and $2.7 \times 10^{-5}$, respectively). The expressions of osteocalcin was significantly higher in the Dex and Dex/AscP group than in the MEM group ( $p=0.003$ and 0.001 , respectively).

The Western blots for collagen type I, laminin and fibronectin are shown in Figure 4. More collagen type I was found in the Dex/AscP group than in the MEM group, whereas the bands of laminin and fibronectin were not increased.
PG measurement is shown in Figure 5. Significantly more proteoglycan was found in the Dex/AscP group than in the MEM group ( $p=0.002)$.

Representative images of the harvested samples of $\beta$ TCP granules combined with the cell sheets at four weeks after transplantation are shown in Figure 6. Macroscopic observation indicated that the transplanted $\beta$-TCP scaffolds aggregated into a single lump in the Dex/AscP group (Fig. 6a). This enhanced bridging bone formation between the $\beta$-TCP scaffolds is most clearly seen in the histological section of the Dex/AscP group (Figs $6 b$ and $6 c$ ).

Figure 7 shows images of the harvested samples of dead femur with $\beta$-TCP granules and cell sheets at four weeks after transplantation. Radiographs show that calcification was observed around the $\beta$-TCP and dead bone in groups treated with $\beta-\mathrm{TCP}$ granules/cell sheets (Fig. $7 b)$. Histological sections of the dead femur with $\beta$-TCP granules/cell sheets created in Dex/AscP showed newly formed bone tissue between $\beta$-TCP granules and the dead femur. In contrast, bone formation was not observed in the gap between $\beta$-TCP granules and the dead femur in the control sample (Fig 7c).

\section{Discussion}

The results of the present study clearly indicate that culture in medium with both Dex and AscP created better osteogenic matrix cell sheets than culture with either Dex or AscP alone. These culture conditions allowed for the mechanical retrieval of the confluent cell layer as a single cell sheet structure that induced bridging bone formation in vivo. The results indicate that culture in Dex/AscP increases cell number and the expression of ECM components such as collagen type I and PG. The biosynthesis and deposition of this ECM by the cultured cells appears to bind the confluent cells into a stable sheet structure. Furthermore, osteoblastic differentiation is induced by the addition of Dex/AscP, which may be advantageous for early bone formation after transplantation. Scaffolds 

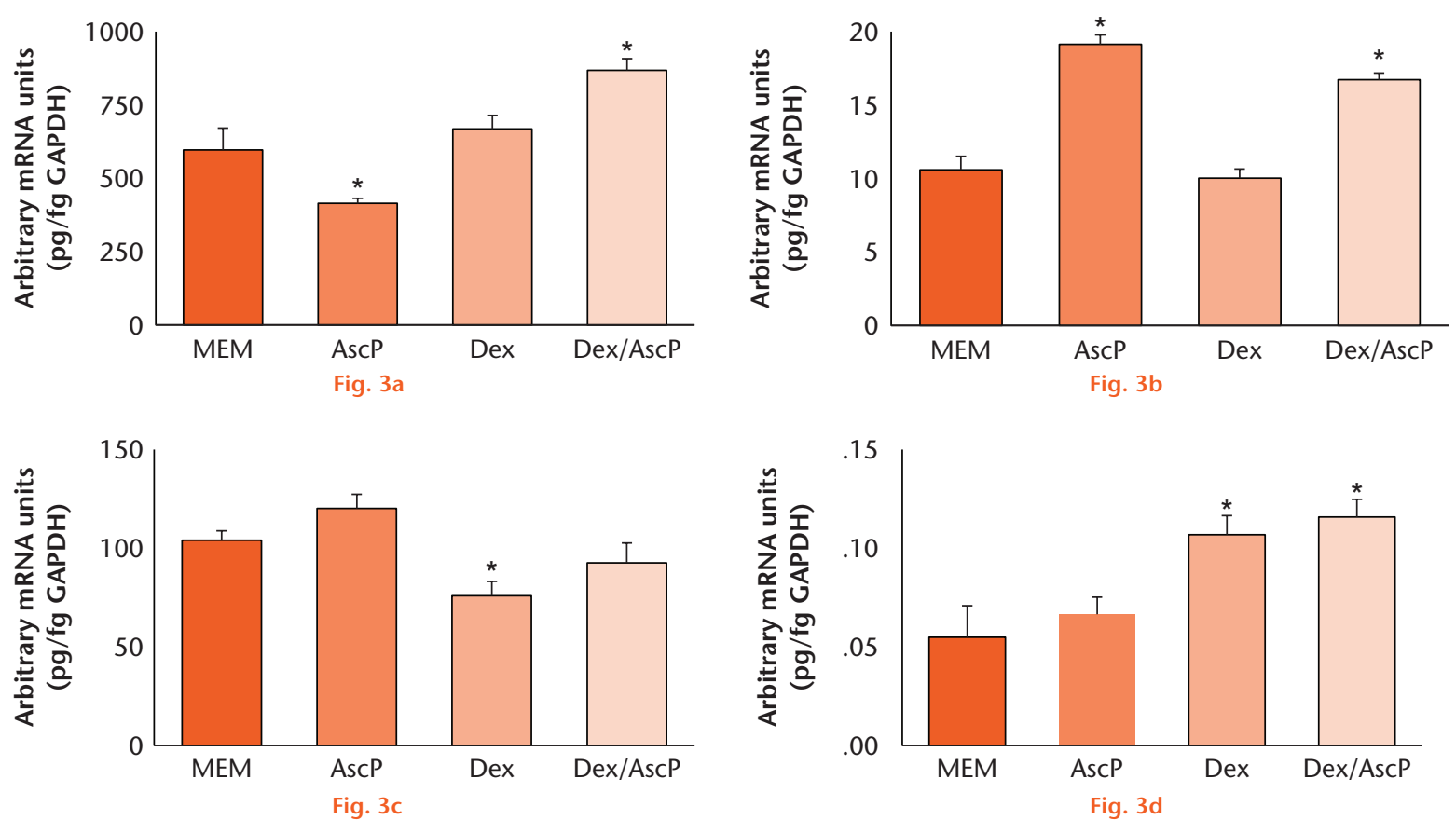

Real-time polymerase chain reaction messenger RNA expression of (A) collagen type I, (B) laminin, (C) fibronectin and (D) osteocalcin.
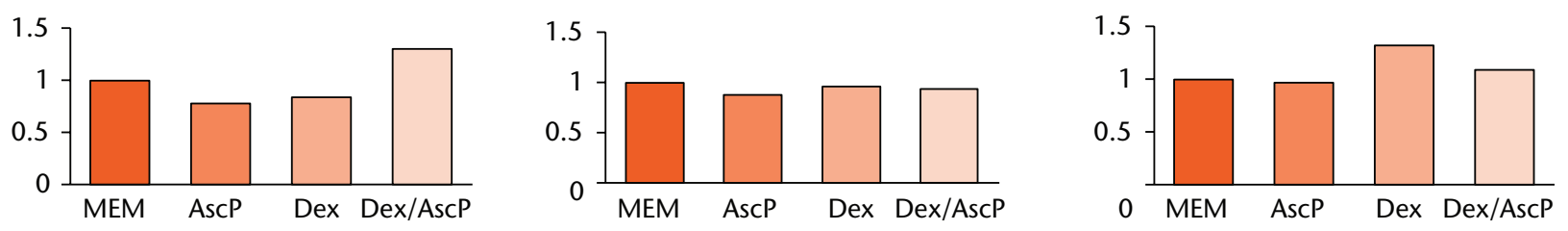

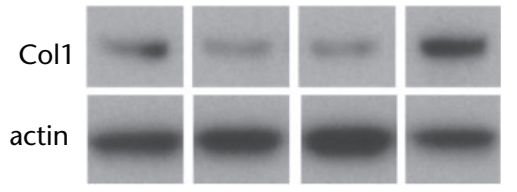

Fig. 4a

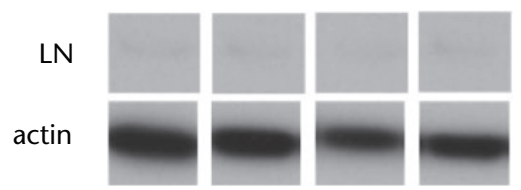

Fig. 4b

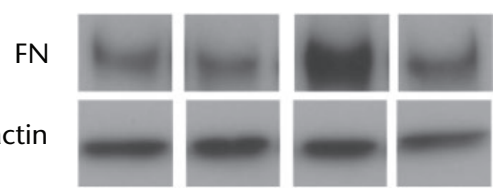

Fig. 4c

Western blots showing that the expression of collagen type I was increased in the Dex/AscP group. The expression ratio of minimal essential medium was defined as 1 unit in the bar figure (upper panel) (Col1, collagen type I; LN, laminin; FN; fibronectin).

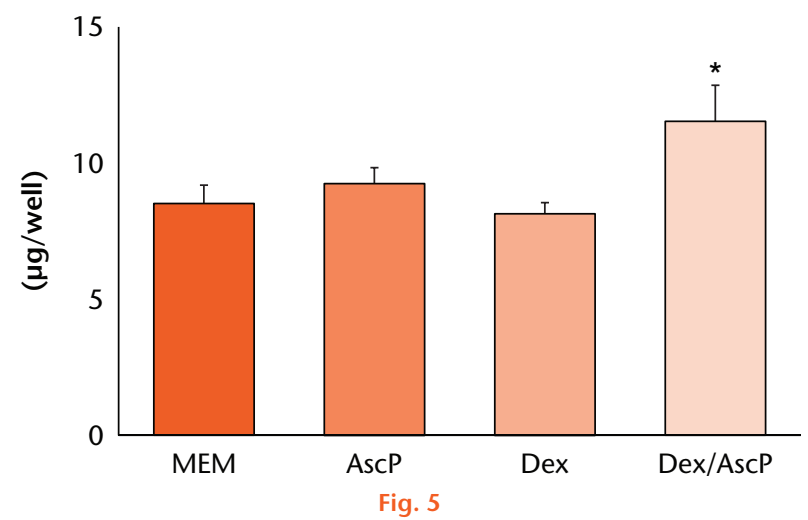

Proteoglycan measurement of the bone marrow stromal cells in the groups ( ${ }^{*} \mathrm{p}=0.002$ ) (MEM, minimal essential medium; AscP, ascorbic acid phosphate; Dex, dexamethasone). are an essential component of tissue engineering methods that play an important role as support structures for cell seeding and growth, as well as promoting tissue repair. ${ }^{30}$ Combining BMSCs with scaffolds, such as $\beta$-TCP or $\mathrm{HA}$, can promote the formation of new bone tissue after subcutaneous transplantation. 2,5,16-18 We also previously reported the osteogenic properties of HA constructs combined with BMSCs ${ }^{1,5}$ and HA scaffolds wrapped with BMSC cell sheets. ${ }^{22}$ Based on these results, we believe that cell sheet transplantation can be a useful method for making tissue-engineered bone for clinical applications.

Collagen has recently received considerable attention as a favourable matrix for seeding cells. Collagen is important for many tissue engineering methods, and is widely used for bone repair and new bone formation 

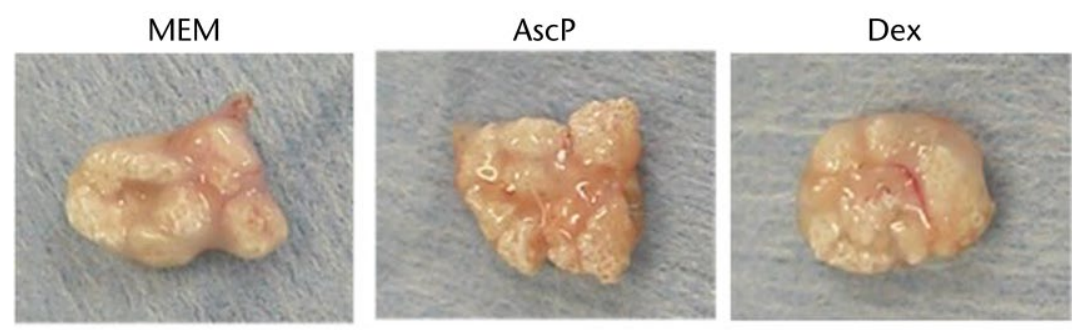

Fig. $6 a$
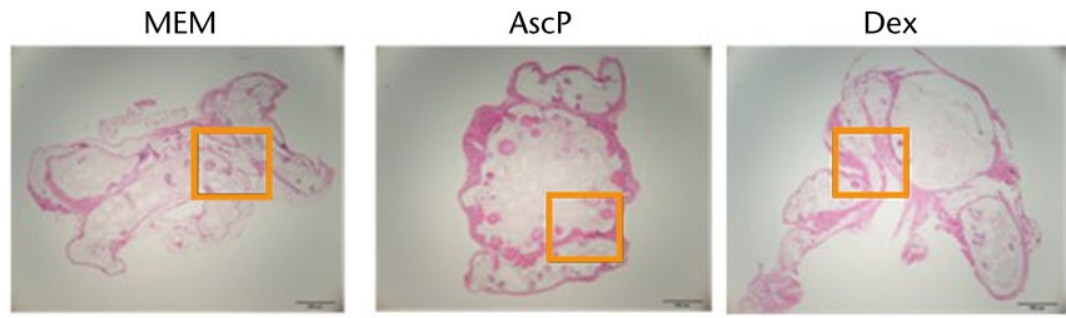

Fig. $6 \mathrm{~b}$
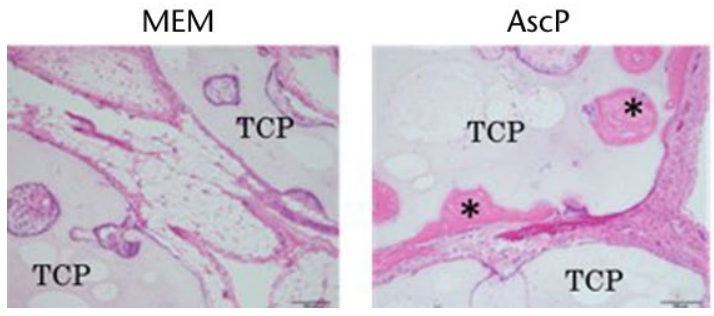

Dex

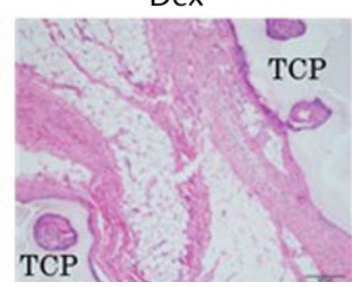

Fig. 6c

Representative images of the samples harvested four weeks after transplantation: a) macroscopic observation indicated that the transplanted $\beta$-tricalcium phosphate (TCP) scaffolds aggregated into a single lump in the dexamethasone (Dex)/ascorbic acid phosphate (AscP) group; b) and c) haematoxylin and eosin stained sections showed remarkable bridging bone formation between the $\beta$-TCP scaffolds in the Dex/AscP group. The panels in (c) are higher magnification views of the rectangular areas indicated in (b) (*bone tissue; bar, $200 \mu \mathrm{m}$ ).

because it is biocompatible, improving MSC survival. ${ }^{31}$ Different types of collagen are the main components of most fibrous connective tissues, such as tendon, ligament, and skin, as well as cartilage and bone. Collagen type I, which consists of two $\alpha 1$ and one $\alpha 2$ chains, is the most abundant protein in mammals, and a natural ECM that supports most tissues and cell structures. It is also present in bone and teeth and is the main organic component of bone. Laminin, which consist of $\alpha-, \beta-$, and $\gamma$-chains, is the major protein component of the basement membrane, which forms a protein network foundation for cells, as well as organs. The laminin that is secreted and incorporated into the ECM belongs to the glycoprotein family and is an essential part of the structural scaffolding in most animal and human tissues. In the present study, culture with Dex/AscP increased cell number and the production of ECM components, including collagen type I and PG, resulting in the formation of an osteogenic matrix cell sheet. Unlike in methods that use trypsin/EDTA digestion, because the ECM in an osteogenic matrix cell sheet remains intact, they are suitable for skeletal reconstruction applications such as bone defects and fracture nonunions. Transplantation of an osteogenic matrix cell sheet includes both necessary features of cell transplantation and ECM transplantation, which together, can fill in gaps between bones and artificial bone, ${ }^{11,12}$ as well as to unite artificial bones. PGs are a major component of the animal extracellular matrix and are formed of glycosaminoglycans (GAGs) covalently attached to the core proteins. PG and GAG side-chains form supramolecular aggregates that interconnect the collagenous network in connective tissues, and play a significant role in regulating the mechanical behaviour of the extracellular matrix, particularly in soft tissues. ${ }^{32}$

The standard procedure for osteogenic differentiation of stem cells is treatment of culture cells with Dex, AscP and $\beta$-glycerophosphate ( $\beta$-GP). Langenbach and Handschel ${ }^{33}$ reported that Dex induces osteogenic differentiation of BMSCs by increasing the expression of mitogen-activated protein kinase phosphatase-1 (MKP-1), which dephosphorylates and activates runt-related transcription factor 2 (Runx2). AscP leads to the increased secretion of collagen type I, which facilitates osteogenic differentiation. Others reported that Dex prevents apoptosis of BMSCs ${ }^{34}$ and promotes MSC proliferation. ${ }^{35}$ In our method of cell sheet fabrication, we cultured BMSCs with Dex and AscP, therefore enhancing osteogenic potential and ECM (collagen type I and PG) production, and resulting in a 

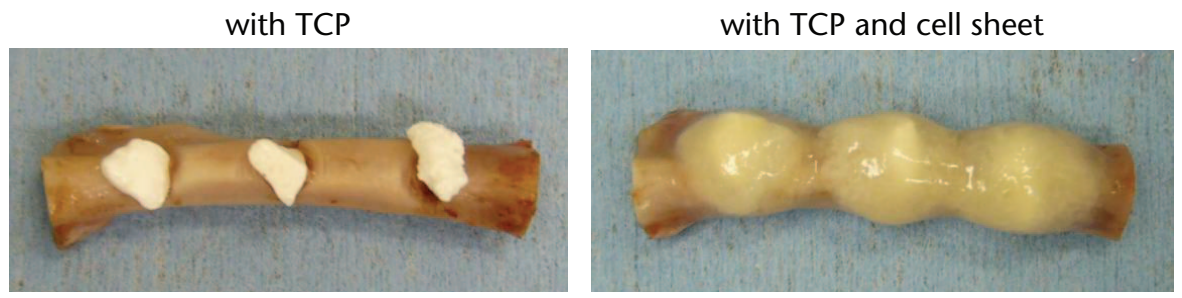

Fig. $7 a$

with TCP

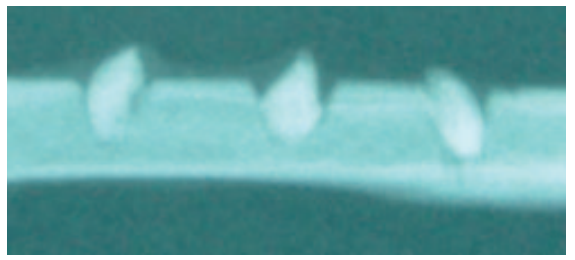

with TCP and cell sheet

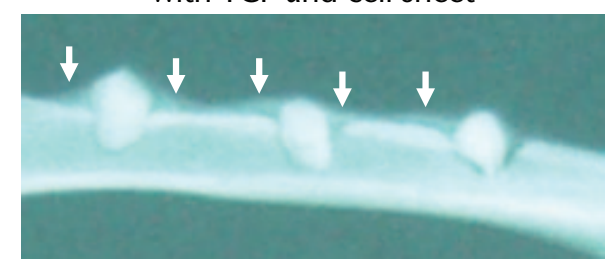

Fig. 7b

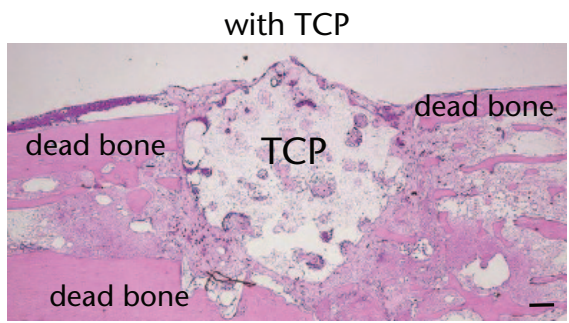

with TCP and cell sheet

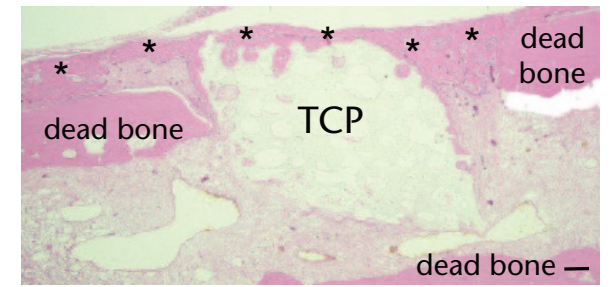

Fig. 7c

Representative images of the dead bone with $\beta$ - tricalcium phosphate (TCP) samples: a) macroscopic appearance before transplantation; b) the radiograph indicates that calcification was observed around $\beta$-TCP in the group treated with $\beta$-TCP granules combined with the cell sheet; $c$ ) haematoxylin and eosin stained sections showed remarkable bridging bone formation between the $\beta$-TCP scaffolds and dead bone in the group treated with $\beta$-TCP granules and the cell sheet created in dexamethasone/ascorbic acid phosphate The white arrow indicates calcification (*newly formed bone tissue; bar: $200 \mu \mathrm{m})$.

complete cell sheet structure and abundant bone formation in vivo.

Recently, many researchers have reported methods for fabricating cell sheets, e.g., using special culture plates or multifunctional copolymers. ${ }^{36-38}$ Specifically, Yang et al ${ }^{38}$ reported that the use of culture dishes coated with thermosensitive polymers, which have been clinically applied for the treatment of corneal surface dysfunction. Other researchers reported on the mechanical retrieval method of cell sheet fabrication by a scraping technique, which formed bone tissue within a subcutaneous site, ${ }^{23}$ consistent with our previous results. ${ }^{22,27}$ However, these other groups created their cell sheet in MEM with Dex, AscP and $\beta$-GP. In our pilot study, it was difficult to scrape off the cell sheet when cells were cultured with Dex, AscP and $\beta$-GP as calcium deposition disturbed the process of smooth scraping (data not shown).

There are a few limitations to the present study. First, the study was performed on rat BMSCs. Because human BMSCs are used in the clinical application of cell sheets created using our technique, human BMSCs should be used to determine whether culture with Dex/AscP improves the integrity of osteogenic matrix cell sheets. Secondly, although we assessed the expression of the ECM components collagen types I, laminin, fibronectin and PG to show the potential mechanism of cell sheet formation, there are many other ECM components that were not assessed. Thirdly, we cultured the BMSCs in only a single concentration of Dex and AscP. To determine the optimal concentrations of Dex and AscP that create stable cell sheets, a range of treatment doses are necessary. However, no obvious tears were found in the sheet structures cultured with Dex and AscP, indicating that the cell sheets may be useful for tissue engineering methods of bone regeneration. Finally, we did not compare the characteristics of cell sheets created using our method with those of cell sheets created by other techniques, such as by using a thermosensitive polymer. Therefore, other techniques for making cell sheets from BMSCs should be similarly tested. Future studies will address these issues.

In conclusion, the data from the present study indicate that culture with Dex and AscP increased the cell number and expression of the ECM components collagen types I and $P G$, resulting in a stable cell sheet structure. These culture conditions allowed for the mechanical retrieval of the confluent cell layer as a single cell sheet structure that induced bridging bone formation in vivo. Because of its clear benefit to cell sheet structure, this method may be beneficial when applied to cases where tissue reconstruction is difficult. 


\section{References}

1. Akahane M, Ueha T, Dohi $\mathbf{Y}$, et al. Secretory osteocalcin as a nondestructive osteogenic marker of tissue-engineered bone. J Orthop Sci2011;16:622-628.

2. Ohgushi H, Caplan Al. Stem cell technology and bioceramics: from cell to gene engineering. J Biomed Mater Res 1999;48:913-927.

3. Tsuji W, Rubin JP, Marra KG. Adipose-derived stem cells: implications in tissue regeneration. World J Stem Cells 2014;6:312-321.

4. Ūsas A, Mačiulaitis J, Mačiulaitis R, et al. Skeletal muscle-derived stem cells: implications for cell-mediated therapies. Medicina (Kaunas) 2011;47:469-479.

5. Akahane M, Ohgushi H, Yoshikawa T, et al. Osteogenic phenotype expression of allogeneic rat marrow cells in porous hydroxyapatite ceramics. J Bone Miner Res 1999;14:561-568.

6. Wakitani S, Imoto K, Yamamoto T, et al. Human autologous culture expanded bone marrow mesenchymal cell transplantation for repair of cartilage defects in osteoarthritic knees. Osteoarthritis Cartilage 2002;10:199-206.

7. Woodbury D, Schwarz EJ, Prockop DJ Black IB. Adult rat and human bone marrow stromal cells differentiate into neurons. J Neurosci Res 2000;61:364-370.

8. Brazelton TR, Rossi FMV, Keshet GI. From marrow to brain: expression of neuronal phenotypes in adult mice. Science 2000;290:1775-1779.

9. Ohishi M, Schipani E. Bone marrow mesenchymal stem cells. J Cell Biochem 2010;109:277-282.

10. Salem HK, Thiemermann C. Mesenchymal stromal cells: current understanding and clinical status. Stem Cells 2010;28:585-596.

11. Ueha $\mathbf{T}$, Akahane $\mathbf{M}$, Shimizu $\mathbf{T}$, et al. Utility of tricalcium phosphate and osteogenic matrix cell sheet constructs for bone defect reconstruction. World J Stem Cells 2015;7:873-882.

12. Uchihara Y, Akahane M, Shimizu T, et al. Osteogenic Matrix Cell Sheets Facilitate Osteogenesis in Irradiated Rat Bone. BioMed Res Int 2015;629:168.

13. Matsushima A, Kotobuki N, Tadokoro $\mathbf{M}$, et al. In vivo osteogenic capability of human mesenchymal cells cultured on hydroxyapatite and on $\beta$-tricalcium phosphate. Artif Organs 2009;33:474-481.

14. Kawate K, Yajima H, Ohgushi H, et al. Tissue-engineered approach for the treatment of steroid-induced osteonecrosis of the femoral head: transplantation of autologous mesenchymal stem cells cultured with beta-tricalcium phosphate ceramics and free vascularized fibula. Artif Organs 2006;30:960-962.

15. Ter Brugge PJ, Jansen JA. In vitro osteogenic differentiation of rat bone marrow cells subcultured with and without dexamethasone. Tissue Eng 2002;8:321-331.

16. Nakamura A, Dohi Y, Akahane $\mathbf{M}$, et al. Osteocalcin secretion as an early marker of in vitro osteogenic differentiation of rat mesenchymal stem cells. Tissue Eng Part C Methods 2009;15:169-180.

17. Bianco P, Robey PG. Stem cells in tissue engineering. Nature 2001;414:118-121.

18. Petite H, Viateau V, Bensaïd W, et al. Tissue-engineered bone regeneration. Nat Biotechnol 2000;18:959-963.

19. Dong J, Kojima $\mathbf{H}$, Uemura $\mathbf{T}$, et al. In vivo evaluation of a novel porous hydroxyapatite to sustain osteogenesis of transplanted bone marrow-derived osteoblastic cells. J Biomed Mater Res 2001;57:208-216.

20. Shimizu T, Sekine H, Yamato $\mathbf{M}$, et al. Cell sheet-based myocardial tissue engineering: new hope for damaged heart rescue. Curr Pharm Des 2009;15:28072814.

21. Kanai $\mathbf{N}$, Yamato M, $\mathbf{0 k a n o}$ T. Cell sheets engineering for esophageal regenerative medicine. Ann Trans/ Med2014;2:28.

22. Akahane M, Nakamura A, Ohgushi $\mathbf{H}$, et al. Osteogenic matrix sheet-cell transplantation using osteoblastic cell sheet resulted in bone formation without scaffold at an ectopic site. J Tissue Eng Regen Med 2008;2:196-201.

23. Ma D, Ren L, Liu Y, et al. Engineering scaffold-free bone tissue using bone marrow stromal cell sheets. J Orthop Res 2010;28:697-702.
24. Akahane $\mathbf{M}$, Ueha $\mathbf{T}$, Shimizu $\mathbf{T}$, et al. Cell sheet injection as a technique of osteogenic supply. Int J Stem Cells 2010;3:138-143.

25. Nakamura A, Akahane M, Shigematsu H, et al. Cell sheet transplantation of cultured mesenchymal stem cells enhances bone formation in a rat nonunion model. Bone 2010;46:418-424.

26. Inagaki $\mathbf{Y}$, Uematsu K, Akahane $\mathbf{M}$, et al. Osteogenic matrix cell sheet transplantation enhances early tendon graft to bone tunnel healing in rabbits. Bio Med Res Int 2013;842:192.

27. Akahane M, Shigematsu H, Tadokoro M, et al. Scaffold-free cell sheet injection results in bone formation. J Tissue Eng Regen Med 2010;4:404-411.

28. Shimizu T, Akahane M, Ueha T, et al. Osteogenesis of cryopreserved osteogenic matrix cell sheets. Cryobiology 2013;66:326-332.

29. Tohma Y, Dohi Y, Ohgushi H, et al. Osteogenic activity of bone marrow-derived mesenchymal stem cells (BMSCs) seeded on irradiated allogenic bone. J Tissue Eng Regen Med 2012;6:96-102

30. Kim HJ, Kim U-J, Vunjak-Novakovic G, et al. Influence of macroporous protein scaffolds on bone tissue engineering from bone marrow stem cells. Biomaterials 2005;26:4442-4452.

31. Oh S-A, Lee H-Y, Lee JH, et al. Collagen three-dimensional hydrogel matrix carrying basic fibroblast growth factor for the cultivation of mesenchymal stem cells and osteogenic differentiation. Tissue Eng Part A 2012;18:1087-1100.

32. Bertassoni LE, Swain MV. The contribution of proteoglycans to the mechanical behavior of mineralized tissues. J Mech Behav Biomed Mater 2014;38:91-104.

33. Langenbach F, Handschel J. Effects of dexamethasone, ascorbic acid and $\beta$ glycerophosphate on the osteogenic differentiation of stem cells in vitro. Stem Cell Res Ther 2013;4:117.

34. Song IH, Caplan Al, Dennis JE. Dexamethasone inhibition of confluence-induced apoptosis in human mesenchymal stem cells. J Orthop Res 2009;27:216-221.

35. Wang H, Pang B, Li Y, et al. Dexamethasone has variable effects on mesenchymal stromal cells. Cytotherapy 2012;14:423-430.

36. Nishida K, Yamato M, Hayashida Y, et al. Corneal reconstruction with tissueengineered cell sheets composed of autologous oral mucosal epithelium. $N$ Engl J Med 2004;351:1187-1196.

37. Kim YS, Lim JY, Donahue HJ, et al. Thermoresponsive terpolymeric films applicable for osteoblastic cell growth and noninvasive cell sheet harvesting. Tissue Eng 2005;11:30-40.

38. Yang J, Yamato M, Nishida K, et al. Corneal epithelial stem cell delivery using cell sheet engineering: not lost in transplantation. J Drug Target 2006;14:471-482.

Funding Statement

M. Akahane reports funding received from the Translational Research Network Program from the Japan Agency for Medical Research and Development (AMED) which is related to this article.

Author Contribution

M. Akahane: Designed the study, Prepared the manuscript.

- T. Shimizu: Assisted in the study processes and preparations.

- T. Kira: Assisted in the study processes and preparations.

T. Onishi: Nara Medical University Faculty of Medicine

- Y. Uchihara: Assisted in the study processes and preparations.

T. Imamura: Assisted in manuscript preparation, Critically reviewed the manuscript.

Y. Tanaka: Assisted in manuscript preparation, Critically reviewed the manuscript.

ICMJE conflict of interest

None declared

(c) 2016 Akahane et al. This is an open-access article distributed under the terms of the Creative Commons Attributions licence (CC-BY-NC), which permits unrestricted use, distribution, and reproduction in any medium, but not for commercial gain, provided the original author and source are credited. 